\title{
Research on Start-up Mark Mechanism and Intelligent Compensation
}

\author{
Zhou Qihong, Zhang Jianan, Shen Qiong, Chen Guangfeng
}

Engineering Research Center of Textile Equipment, Ministry of Education, Donghua University, Shanghai, 201620, China

Email: zhouqihong@dhu.edu.cn

Keywords: Start-up Mark; Intelligent Compensation; loom; weaving.

\begin{abstract}
Start-up mark is an important factor leading to woven defects. The reasons of start-up marks were discussed. At present, cloth fell displacement is a main factor to produce start-up marks for a loom. The relationship between cloth fell displacement and its influence factors were studied. Intelligent start-up mark compensatory system based on fuzzy logic was discussed. The fuzzy reasoning algorithm of this system can automatically calculate the compensatory value of start-up mark based on three parameters including the shut-down time of loom, yarn count and yarn variety. This system makes settling start-up mark problem easier and finds a new way to deal with the start-up mark. Finally, the system put forward the compensatory method of start-up mark. This system has been applied to loom control successfully.
\end{abstract}

\section{Introduction}

In the textile system, start-up mark is very complicated and important to fabric quality, and textile enterprises often loss a lot because of start-up mark problem. The solution level of start-up mark directly determines the grade of loom. There are all kinds of reasons of start-up mark discussed in these literatures [1-5], such as decreased motion precision of loom because of the worn beating-up mechanism, the first insufficient weft beating-up force, the low foot frame rigidity, the uneven spindle speed in the first weft cycle, or the delayed brake. The above-mentioned causes of start-up mark can be avoided due to excellent performance of modern looms. For example, modern loom makes the first weft beating-up force close or equal to normal value by adopting super starting torque motor[6].

Cloth fell position deviation caused by creep and relaxation properties is the main reason for start-up mark. As new fabric products especially thin high-grade special fabric are on developing, start-up mark problems loom is still in the continuous research. This thesis mainly studies start-up mark mechanism caused by the loom cloth fell position and the intelligent compensation, to reduce artificial operation difficulty and raise the solution level of start-up mark compensation.

\section{Start-up Mark Mechanism}

\section{Movement Analysis of Cloth Fell Position}

Because fabric and warp are viscoelastic, they are under the larger tension when the loom stop. Then fabric and warp have creep and relaxation .Cloth fell position is moved forward or backward and deviated from its normal position. when the loom start again, first weft yarn is failed to fully enter into the woven to form a sparse mark, or to form a dense mark because of the previous too close weft yarn. The mechanism of start-up mark is analyzed of cloth fell position deviation caused by creep and relaxation properties of fabric and warp, to provide theoretical guidance for the research of start-up mark compensation.

The fabric and warp tension is constant and smaller, and the tension of them is equal when the loom with active back rest and adjusting let-off tension mechanism stops. There is the fabric elastic mechanics model [7] shown in figure 1 if cloth fell position movement is only considered. So the cloth fell position movement is creep process of fabric under constant and small tension for this kind loom. The creep model and its mechanism of cloth fell position shifting were analyzed as 
follows.

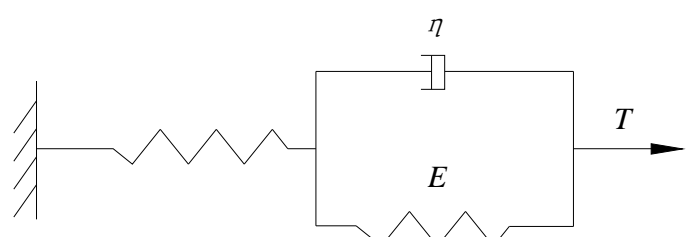

Fig.1. Fabric delayed elastic mechanics model

The tension of the elastic part is the sum of the elastic force and damping force, namely:

$T=T_{\varepsilon}+T_{\eta}$

According to hooke's law and Newton's law of viscosity, the elastic force and damping force of low elastic part are as follows:

$$
\begin{aligned}
& T_{\varepsilon}=\frac{\delta E}{l} \\
& T_{\eta}=\eta \frac{d}{d t}\left(\frac{\delta}{l}\right)
\end{aligned}
$$

Where in (2),(3): $E$ is the modulus of elasticity for fabric delayed elastic part. $\eta$ is the viscous coefficient for fabric delayed elastic part. $l$ is the fabric length factor whose value is multiples of 1 m long fabric.

According to type(1),(2) and (3), (4) is available as follows:

$$
T=\frac{\delta E}{l}+\eta \frac{d}{d t}\left(\frac{\delta}{l}\right)
$$

After deformation, (5) is available:

$$
\frac{d \delta}{T l / E-\delta}=\frac{E}{\eta} d t
$$

To have integrals on both sides at the same time, (6) is available:

$$
-\ln \left(\frac{T l}{E}-\delta\right)=\frac{E}{\eta} t+c
$$

The fabric tension is small when the loom is on beating-up. To be simplicity, beating-up tension is set to zero and when beating-up begin is starting time. Namely $t=0, \delta=0$, so the integral constant $c$ is as follows:

$$
c=-\ln \left(\frac{T l}{E}\right)
$$

Handle equation (7) into equation (6), delayed elastic deformation namely cloth fell position displacement is as follows:

$$
\delta=\frac{T l}{E}\left(1-\frac{1}{e^{E t / \eta}}\right)
$$

Deformation $\delta$ is delayed elastic deformation and irreversible. It can lead to cloth fell position deviation to form a thin or dense weft fabric. From the equation above, we can conclude that the size of the delayed elastic deformation $\delta$ is proportional to the tension and length of fabric and exponential to shut-down time of loom. At the same time, the size of the delayed elastic deformation is also connected with the elastic modulus and coefficient of viscosity. And elastic modulus and coefficient of viscosity are changing with the serial number and density of warp and weft yarn, fabric weave, warp sizing rate, as well as the air temperature and humidity of workshop.

\section{Cloth Fell Position, Beating-up Force and Start-up Mark}

The relationship among cloth fell position and beating-up force and Start-up mark is analyzed. According to the beating-up theory put forward by the Greenwood [8] [9], the weft spacing $S$ can be 
expressed by beating-up force $F$ and cloth fell displacement $L$ when the reed beats up to the top position:

$$
\begin{aligned}
& F=\frac{k}{S-D} \\
& L=\frac{K}{S-D}+S
\end{aligned}
$$

where in (10):

$$
K=\frac{k}{E_{1} / l_{1}+E_{2} / l_{2}}
$$

where in (9),(10),(11): $F$ is beating-up force, $k$ is weaving drag coefficient, $S$ is weft spacing, $D$ is theoretical minimum weft spacing, $L$ is the cloth fell displacement (taking the most forward position of reed beating-up as zero reference point, forward from loom as positive, backward from loom as negative), $E_{1}$ is warp elastic modulus, $E_{2}$ is fabric elastic modulus, $l_{1}$ is free length of warp, $l_{2}$ is free length of fabric.

To have differentials on (9) and (10), the following are available:

$$
\begin{aligned}
& d S=\frac{(S-D)^{2}}{k} d F \\
& d F=\frac{k}{(S-D)^{2}+K} d L
\end{aligned}
$$

According to (12) and (13),dynamic location change of cloth fell in each revolution of the spindle is regular during normal operation. The relative position of cloth fell remains unchanged in the beginning of each beating-up, namely $d L=0$. While at the end of beating-up, maximum weaving resistance which is from cloth fell to reed is constant. As a result, the movement amount of weft around cloth fell is normal relative to the warp under the action of the reed and weft density is uniform. So there will be no set marks. Once cloth fell position is deviated forward from its normal position, namely $d L>0$, beating-up start time delayed, then $d F<0$, meaning beating-up force drops, a sparse mark appears on the fabric. If cloth fell position is deviated backward from its normal position, namely $d L<0$, beating-up start time advanced, then $d F>0$, meaning beating-up force increases, and a dense mark appears on the fabric. Therefore the change of cloth fell position leads to the change of beating-up force, and the change of beating-up force eventually causes weft density unevenness. Then motion mark is formed.

It is known from the above analysis cloth fell position is deviated from its normal position due to creep and relaxation of fabric and warp after loom stoppage. when loom start again, the offset of cloth fell position leads to the change of beating-up force, and the change of beating-up force eventually causes weft density unevenness, thereby start-up mark is formed.

\section{Fuzzy Intelligent Reasoning of Start-up Mark Compensatory Value}

The integrated let-off and take-up control system by electronic let-off and electronic take-up is easier to eliminate start-up marks. We can find solution from let-off and take-up equipments. In order to reduce the warp creep, to relax the warp in the shut-down process and then tighten the warp while driving it is taken. But in practice, this method is not desirable. It increases the difficulty of the control system. Maybe creep decreased, but the process of the yarn relaxation and tightening will certainly cause the movement of cloth fell position. The displacement is affected by many factors. It increases the difficulty of the start-up mark solution in turn. So it is rarely used in practice. For slow motion, changing the corresponding speed of let-off and take-up motion can compensate for the shortage of beating-up forces. Start-up mark problem caused by loom stop can be solved by compensation of yarn displacement after starting. This is also an effective method widely used now. The key is to determine the compensatory value to meet requirements of the different species and shut-down time.

How to set specific compensatory value of cloth fell is the key of start-up mark compensation. 
Trial and error method is mainly adopted in domestic electronic let-off and take-up system. There is no theoretical guidance to set start-up mark in general textile enterprises. It's hard to adjust to the optimal degree coupled with the relatively low level of knowledge of textile workers. Debugging work will greatly affect production efficiency. It often cost worker more than an hour to try once. Moreover in order to get an ideal value it is need to debug it repeatedly. There is no way even to adjust to satisfactory condition.

The main reasons of start-up mark formation was theoretically analyzed above. A set of simple and practical start-up mark intelligent compensatory system is discussed based on the idea of fuzzy reasoning. In the system users only need enter two parameters simply rather than blindly debug.

Fabric tension and fabric length are all relatively fixed constant for most fabric in textile workshops. So the major influencing factors of cloth fell position are loom shut-down time, yarn elastic modulus and yarn variety coefficient. The actual situation in several workshops show that environmental temperature and humidity are under the similar circumstances, some of the above parameters are mainly decided by warp thickness degree(count) and yarn variety. Other parameters have little effect. This is also consistent with the previous theoretical analysis. If compensatory value can be acquiredaccording to the several parameters, start-up mark problem will be solved easily. Users are familiar with these parameters.

How these three parameters in the end affect start-up mark is qualitative. The system is nonlinear. The various factors coupled to each other, and it is not easy to be modeled. Fuzzy mathematics is very effective to solve such problems [10-11]. Therefore the fuzzy reasoning algorithm is adopted to automatically calculate the compensatory value of start-up mark. On the basis of the compensatory value calculated, if it still can't meet the requirements, users just need fine-tuning to quickly get the actual compensatory value of the system. The operation is simple, and greatly reduces the debugging time.

The fuzzy reasoning algorithm of this system can automatically calculate the compensatory value of start-up mark based on three parameters including the loom shut-down time, yarn count and yarn variety. The three parameters have a greatest impact on start-up mark. The longer the loom shut-down time, the finer the yarn and the smaller the yarn elastic modulus, the greater the displacement of cloth fell position. Conversely, the smaller the displacement of cloth fell position. The system uses fuzzy algorithm to realize fuzzification of inputs including the loom shut-down time, yarn count and yarn elastic modulus. The fuzzy output can be calculated according to fuzzy control rules. Then the displacement of cloth fell position can be automatically calculated by defuzzification.

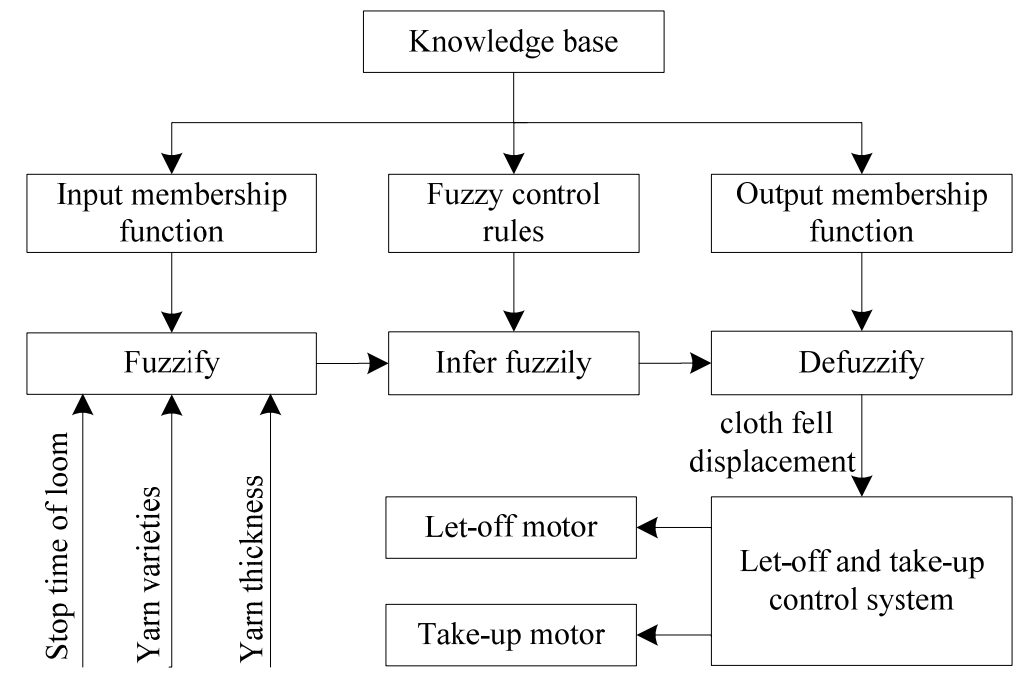

Fig.2. Fuzzy compensation principle of start-up mark

Users simply need enter only warp count and yarn variety parameters. The system automatically calculates shut-down time after loom stoppage. Then the system calculates compensatory value according to the three parameters. Then system makes compensation according to the compensation 
mode and setting weft count. The position and speed of the let-off and take-up motor is adjusted to meet the control requirement.

It should be noted that sometimes loom is set to stop at different angles when warp break and weft break happen for the convenience of operation. Loom stop in the position where the warp tension is low when warp break happens. Warp deformation is small. Loom stop in the position where the warp tension is high when weft break happens. So the deformation is different. The deformation for weft break is bigger than the deformation for warp break. The specific value of displacement is proportional to tension of shut-down time according to the previous theoretical analysis of cloth fell displacement. So the system will automatically determine different compensatory values according to stop type.

\section{Driving Compensatory Method of Cloth Fell Position}

It is equally distributed to a few values according to different compensatory method after the compensatory value is calculated. Linear compensation, absolute value compensation, exponential compensation are often adopted. Principle of linear compensation is shown in figure 3.

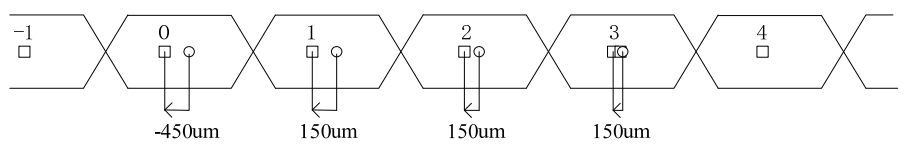

$\square$ With compensation $\bigcirc$ Without compensation

Fig.3. Schematic Diagram of Start-up Mark Compensation

Zero point in the above figure is shut-down position. Points mark with $\bigcirc$ are uncompensated position. Points mark with $\square$ are compensated position. The result of compensation is to make the displacement deviation to zero. The weft number of compensation is determined by experiences and experiments. Generally the number is no greater than 5 .

\section{Conclusion}

In this study various factors of start-up mark are analyzed. It is concluded that cloth fell displacement of new type loom is the main factor causing start-up mark. The relationship between cloth fell displacement and its influence factors are analyzed theoretically which provides the theoretical guidance for compensation of start-up mark. The intelligent compensation system of start-up mark is developed preliminary based on fuzzy reasoning. The compensatory system can calculate the compensatory value of start-up mark according to yarn variety and count, which greatly reduces the debugging difficulty of start-up mark compensation. The relation of compensatory system between warp break stop and weft break stop is also studied in theory. It settles the existent problem in manufacturing. Finally, the system put forward the compensatory method of start-up mark. This system has been applied to loom control successfully.

\section{Acknowledgement}

In this paper, the research was supported by the Fundamental Research Funds for Central Universities (Project No.15D110314, No.15D110317 and No.15D110316).

\section{References}

[1]G. Chen, Y. Wang. A low-tension method for start-up mark prevention in weaving. The Journal of The Textile Institute.2007, Volume 98, Issue 1, 81-85.

[2]Premakumara Ranishka,Perera Thanura,Prince Stephan,Fernando E.A.S.K.Mathe-matical analysis of start-up mark in elastic tape on a narrow fabric loom. Moratuwa Engineering Research Conference. May 22, 2015, 50-54, . 
[3]Wu Hefu, Mark of Drive Rapier Loom, Chemical Fiber \& Textile Technology.March,2014,Vol. 43, No. 1, 37-41.

[4]Sun Yonggang, Zhang Zhenhua, Qu Jinling. et al. Textile Accessories. My tentative view on the stripiness of the cloth produced by the air-jet loom. 2012,November, Vol. 39 No.6, p35-37.

[5]Sun Yu. Shandong Textile Science \& Technology. Analysis on the Causes of K88 Rapier Loom Defects \& Discussion on the Solved Measures.2010(03), 15-17.

[6]Li Xue,CaiYan,WenYanbin. Journalof Tianjin Polytechnic University. Control system of eliminating loom start-up marks based on switched reluctance motor.2012,April,Vol.31 No.2,82-85.

[7]Laizhen. Research on the Influence of Shedding Motion on Warp Weaving Trait [J]. Silk,2005,(6):40-43.

[8]Greenwood. The Position of The Cloth Fe11 in Power Looms, Part III: Experiment[J].Journal of Textile Institute.1956, 47:T274-286.

[9]K. Greenwood , W.T.Cowhig. The Position of The Cloth cell Looms. Part I : Stable Weaving Conditions[J].Journal of Textile Institute in Power.1956 (47):241-254.

[10]Precup, Radu-Emil; Hellendoorn, Hans; A survey on industrial applications of fuzzy control. Source: Computers in Industry. April 2011,62(03), 213-226.

[11]Mohammad, Mehdi Fateh, Siamak Azargoshasb. Nonlinear Dynamics Discrete adaptive fuzzy control for asymptotic tracking of robotic manipulators. November 2014, Volume 78, Issue 3, 2195-2204. 